Pub. Mat. UAB

№ 19 Maig 1980

Actas II Congreso de Ecuaciones Diferenciales y

Aplicaciones -valldoreix, Mayo 1979.

\title{
ESPECTRO EN PROBLEMAS INTEGRODIFERENCIALES LIGADOS A LA VISCOELASTICIDAD
}

\author{
Miguel Lobo Hidalgo \\ Universidad de Santiago de Compostela
}

INTRODUCCION.-

Los materiales viscoelásticos son aquellos materia les tales que en su ecuación constitutiva, interviene no solo el vector gradiente del desplazamiento en el instante actual $t$, sino tambien la historia de dicho deplazamiento.

La forma que toma el tensor de deformación, $\sigma_{i j}$, en los materiales viscoelasticos, en el caso de viscoelasticidad lineal, tipo Boltzman, y restrigiendonos, a la dimension de espacio, $n=1$, es

$$
\sigma=c u_{x}-\int_{-\infty}^{t} g(t-\tau) u_{x}(\tau) d \tau
$$

y su ecuación del moviriento viene dada por la ecuación intẹ gro-diferencial

$$
p \ddot{u}=\sigma_{x}
$$

donde $u(x, t)$ es el vector desplamiento, y o la densidad del cuerpo. Cumpliendo la función $g(\xi)$ del integrando, unas cier tas propiedades de convexidad, $y$ decrecimiento en el infini to.

$$
\text { C. Dafermos, en sucesivos trabajos que van de } 1969
$$

a 1975, ha estudiado la ecuación (2) sometida a condiciones iniciales y unicidad de soluciones, asi como a la estabilidad asintótica de las mismas. 
En concreto el problema de la deformación de un material viscoelastico unidimensional, que ocupa el segmento $[a, b], y$ sujeto en sus extremos, se escribe como sigue:

$$
\begin{aligned}
& \rho \ddot{u}=c_{u x}-\int_{-\infty}^{t} g(t-\tau) u_{x x}(\tau) d \tau \\
& u(x, t)=\phi(x, t) \quad t \in(-\infty, 0] \\
& u(a, t)=u(b, t)=0
\end{aligned}
$$

Los sucesivos tratamientos del problema (3) que el propio Dafermos ha utilizado, y que han liegado a nuestro conocimiento, son los siguientes:

1) Reducción a una ecuación integro-diferencial de tipo Volterra.

2) Aplicación de la teoria de semigrupos de contracción.

Hablemos brevemente de cada uno de ellos.

\section{Reducción a una ecuación de Volterra.}

Este tratamiento consiste Gnicamente en sustituir en el integrando de la primera ecuacion de (3), uxx $(\tau)$ por su valor a partir del dato "espeso", $\phi(\tau, x)$, obteniendose asi la ecuación no homogênea de volterra (4).

$$
\rho \ddot{u}=c u_{x x}-\int_{0}^{t} g(t-\tau) u_{x x}(\tau) d \tau+f(t)
$$

$\operatorname{con} f(t)=-\int_{-\infty}^{0} g(t-\tau) u_{x x}(\tau) d \tau$

$y$ dicha ecuacion (4) sometida a las condiciones iniciales

$$
u(0)=\phi_{0}(x), \quad u(0)=\phi_{1}(x)
$$

dond e :

$$
\phi_{0}(x)=\phi(0, x) \quad \phi_{1}(x)=\phi_{t}^{2}(0, x)
$$


La ecuación (4) con las condiciones iniclales (5), no es mas que la version infinito-dimensional de la ecuación de Volterra clasica:

$$
\rho u^{*}=-c u+\int_{0}^{t} g(t-\tau) u(\tau) d \tau \quad t \in[0, \infty)
$$

y que ha sido estudiada sucesivamente por Volterra Friedman Shinbrot [€], [7], Levin y, Nohel, [8], [9], y otros. Remiti mos para una información bibliográfica complementarios a libro de Hale [11].

$$
\text { Dichos trabajos, vienen a estudiar las condicio- }
$$
nes minimas que debe cumplir $\mathrm{g}(\xi)$, $g: P^{+} \rightarrow \mathrm{R}$ para garantizar la estabilidad asintótica de la solución a partir de la construcción de una oportuna función de Liapunov, bien mediante têcnicas ligadas a la transformación de Laplace.

Inspirado en estos trabajos, y fundamentalmente en el de Levin [9], Dafermos deduce desde un punto de vista matemático, el efectode amortiguación, en la deformación de un cuerpo viscoelastico, debido a la inclusión del térnino integral en la ecuación de ondas.

Asi en su trabajo de 1970, Dafermos $|3|$ obtiene como aplicación del teorema de las proyecciones de bions, existencia, unicidad, asi como estabilidad asintótica de las soluciones mediante la construcción de una conveniente función de liapunov.

$$
\text { Dicha construcción que asegura la estabilidad, }
$$
viene condicionada a una hipotesis complementaria, que resulta necesario afadir a las de convexidad y decrecimiento de la funcion positiva $g(\xi)$, y que consiste en lo siguiente:

$$
c-\int_{0}^{\infty} g(\xi) \mathrm{d} \xi>0
$$

Esta condición (7) lleva a una interpretación mecanicista muy clara. Consideremos las soluciones estaciona- 
rias del problema integro-diferencial no homogéneo

$$
\rho \frac{\partial^{2} u}{\partial x^{2}}=c \frac{\partial^{2} u}{\partial x^{2}}-\int_{-\infty}^{t} g(t-\tau) \frac{\partial^{2} u}{\partial x^{2}} d \tau+f(x)
$$

$y \operatorname{con} u(x, \tau)=u(x) \quad \forall \tau \in(-\infty, t]$.

$$
\text { Esta solución satisface a la ecuación }
$$

$$
\left(c-\int_{0}^{\infty} g(\xi) d \xi\right) \frac{\partial^{2} u}{\partial x^{2}}=f(x)
$$

y la ecuación (7) establece simplemente que el "módulo estâ tico de elasticidad" es postivo.

2. Aplicación de la teoría de semigrupos.

otra aproximación al problema que, estamos tratando, consiste en recurrir a la teoria general de semigrupos de contraccion, Dafermos [5].

La orientación es la siguiente: Es posibie obtener una formulación débil del problema (3) en términos de una ecuación de evolución del tipo

$$
\begin{aligned}
& \frac{d u}{d t}=A u \\
& u(0)=u_{c}
\end{aligned}
$$

en un espacio de Hilbert, $H$, donde $A$ es un operador maximal disipativo, y por tanto generador de un semigrupo de cont tracción $\{T(t)\} t \geqslant 0$, donde $T(t) u_{0}$ es la soluciôn finica del prohlema ( 9 ); y por tanto consideramos la estabilidad asintótica de la solución dentro del comportamiento as intótico de dicho semigrupo.

En efecto: Basta introducir una variable auxiliar en la ecuactón $(3), w(x, \xi, t)=u(x, t-\xi), \xi \in R^{+}$; es decir, Ilamamos $w$ a la historia del desplazamiento, y si denotamos 
por $v(x, t)=\dot{b}(x, t)$ la velocidad, la primera ecuación de puede ser escrita en la forma:

$$
\begin{aligned}
& \dot{u}=\rho^{-1} v \\
& \dot{v}=c u_{x x}-\int_{0}^{\infty} g(\xi) w_{x x} d \xi \\
& \dot{w}=-\frac{\partial w}{\partial \xi}
\end{aligned}
$$

y si considereamos al operador integro-diferencial del segundo miembro de (11) en el espacio de Hilbert

$$
H=H_{0}^{1}(\Omega) \times L^{2}(\Omega) \times L_{g}^{2}\left(R^{+} ; H_{0}^{1}(\Omega)\right)
$$

donde indicamos por $\mathrm{L}_{\mathrm{g}}^{2}$, al espacio de las funciones de cuadrado integrable con peso g. Si dotamos al espacio $\mathrm{H}$, de un producto escalar equivalente, donde va a intervenir la condición (7); entances al cierre de dicho operador en $H$, nos lieva a la definición de un operador $A: D(A) \rightarrow H, \overline{D(A})=H$ y $A^{-1} O=\{0\}$, concretamente

$D(A)=\left\{(u, v, w) \in H, v \in H_{0}^{1}(\Omega), \frac{\partial w}{\partial \xi} \in L_{g}^{2}\left(0, \infty ; H \frac{1}{0}(\Omega)\right), w(\cdot, 0)=u(\cdot)\right\}$

Ademâs se verifica, no sin dificultad:

$$
\langle A(u, v, w),(u, v, w)\rangle \leq 0
$$

$$
R(I-A)=H
$$

Es decir, que A es maximal disipativo y por tanto generador de un semigrupo de contracción, $T(t)$, cuyo comportaniento es preciso estudiar.

Una manera de abordar el probletha consiste en demostrar, que el conjunto w-limite $\omega(\phi)$, para cualquiera de sus orbitas $\gamma(\phi)$ con $\phi \in D(A)$ esta formado por un solo punto, y ese punto es el OeH. 
Bajo la condición de que $\mathrm{A}^{-1} 0=\{0\}$, que verifica nuestro operador, es decir que el dnico punto fijo del semi grupo $f(t)$, es el 0 , el problema esta resuelto en Brezis [1], para el caso en que $-A$ es la subdiferencial de una fun ción convexa s.c.i., propia, con una hipatesis complementaria que omitimos.

Por otra parte Dafermos-Slemrod [4], han tratado el problema para operadores maximales disipativos, bajo la hipótesis de que el canjunto w-limite, para las orbitas del semigrupo, sea no vacio; condicion que se verifica si las órbitas son precompactas. Este ultimo hecho viene ligado a su vez al comportamiento del operador resolvente $(I-A)^{-1}$ : La compacidad de dicho operador garantiza la precompacidad de las orbitas.

Asi, la aplicación de estos resultados a nuestro operador, siempre teniendo en cuenta que $\mathrm{A}^{-1}{ }^{\prime} 0=\{0\}$, permite demostrar que en efecto, el conjunto w-limite para cada una de sus orbitas $Y(\phi)=\{0\}, y$ for tento la estabilidad asinto tica de las soluciones. Unicamente cabe mencionar de manera especial, que la compacidad de las orbitas para nuestro pro blema, hay que demostrarla directamente, porque el operador resolvente, $(I-A)^{-1}$ no es compacto en $H$.

OBSERVACION. En $|5|$ se demuestra que fT(t)\} restringido al conjunto $\overline{\operatorname{cow}(\phi)}$, coincide con un grupo lineal de isometrias, por lo cual la relación

$$
\langle A x, X\rangle=\frac{1}{2} \frac{d}{d t}|| T(t) x||^{2}=0
$$

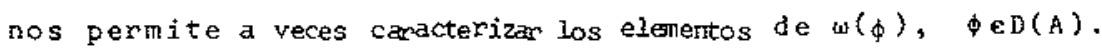

\section{Espectro de $A$.}

Para finalizar, permitasenos contar una pequeña aportación al problema de la viscoelasticidad. 
Puesto que la resolvente del operador A, y por tanto del operador maximal acretivo, $-A$, no es compacta, cono es su espectro.

Es conocido, que la parte puntual de dicho espectro, si existe, esta ligada a las frecuencias propias de vi braciôn de un cuerpo, o tambiên, desde otro punto de vista, a las soluciones de tipo exponencial.

$$
\text { Bien, pues una aproximación a dicho problema es }
$$

la siguiente:

a.- Consideremos el problema de perturbaciones singulares siguiente:

$$
I_{\varepsilon}, \text { en } H \mid \begin{aligned}
& \frac{d \psi}{d t}=A_{\varepsilon} \psi \\
& \psi(0)=\psi_{0}
\end{aligned} \quad A_{\varepsilon} \psi=\left\{\begin{array}{l}
\rho^{-1} v \\
c_{x x}-\varepsilon \int_{0}^{\infty} g(\xi)_{w x} d \xi \\
-\frac{\partial w}{\partial \xi}
\end{array}\right.
$$

$\operatorname{con} \psi=(u, v, w)$

Como probiema reducido consideramos

$$
I_{0} \text {, en } H_{0}=H H_{0}(\Omega) \times L^{2}(\Omega)\left|\begin{array}{l}
\frac{d \theta}{d t}=A_{0}^{\theta} \\
\theta(0)=\theta_{0}
\end{array} \quad A_{0} \theta=\right| \begin{aligned}
& \rho^{-1} v \\
& c u_{x x}
\end{aligned}
$$

$\operatorname{con} \theta=(u, v)$; es decir el problema mixto de condiciones ini ciales para la ecuación de ondas.

No es dificil demostrar la convergencias de soluciones del problema $I_{E}$ a $I_{0}$, en $L^{\infty}\left(0, T ; H_{0}\right)$.

b. - Hay razones para pensar, por tanto que el operador $A_{E}$ cuyo espectro desconocemos admite espectro puntual, próximo al espectro, discreto, del operador antiautoadjunto $A_{0}, y$ cuyos valores propios estan todos en el eje imaginario, denotandolos por $\left\{ \pm i b_{k}\right\} \quad b_{k} \rightarrow \infty$, si $k \rightarrow \infty$. 
En efecto, si nosotros queremos calcular los valo res propios de ${ }_{\varepsilon}$ directamente, nos encontramos con una ecuación del tipo

$$
\zeta^{2}+b_{k}^{2}\left(1-\varepsilon \int_{0}^{\infty} g(\xi) e^{\zeta \xi} d \xi\right)=0 \quad k=1,2, \ldots
$$

donde se ha supuesto $c=1,0=1, \int_{0}^{\infty} g(\xi) d \xi<1$.

La manipulación de dicha ecuación, nos lleva a las siguien tes conclusiones:

1) Si $g(\xi)$ es de tipo exponencial.

$$
g(\xi) \leqslant \mu e^{-\lambda \xi} \quad, \quad \xi \leqslant \frac{\lambda}{2}
$$

el espectro de ${ }_{\varepsilon} A_{\varepsilon} \quad \varepsilon>0$ suficientemente pequeno consta de las siguientes partes.

a) El conjunto $\left\{\zeta \in C, R_{e} \zeta \frac{1}{2}\right\}$ constituye lo que se llama el espectro residual de $A_{\varepsilon}$

$$
\left.\overline{R\left(A_{\varepsilon}\right.}+\zeta\right) \neq H
$$

b) Existen valores propios complejos $\zeta_{k}(\varepsilon)$, próximos a ib $k$, y a -ibk, y no existen valo res propios reales.

c) Los puntos $\zeta e c, \mathrm{R}_{\mathrm{e}} \zeta<\frac{\lambda}{2}$, salvo el espectro puntual $\sigma_{p}$, constituyen el conjunto resolvente $o\left(A_{E}\right)$.

d) Estos resultados pueden hacerse extensivos a $\varepsilon e(0,1\}$ por un argurento del tipo de la invariancia del grado topológico de Brouwer.

Si $g(\xi)$ no fuese de tipo exponencial todo el conjunto de los $\varepsilon C, R_{e}>0$, pertenecen al conjunto residual, y sin interés, parece ser, desde el punto de vista físico. 


\section{BI BLIOGRAFIA}

1. BREZIS, Operateurs Maximaux Monotones. North Holland (1973).

2. DAFERMOS, Asymptotic Stability in Viscoelasticity. Arch. Rat. Mech. ANalysis, 37 (1970), 297-308.

3. DAFERMOS, An Abstract Volterra equation with applications to linear viscoeslaticity. J. Diff. Eqs. 7 $(1970), 554-569$.

4. DAFERMO?-SLEMROD, Asymptotic behavior of nonlinear contraction semigroup. J. Functional Analysis, $13(1973), 97-106$.

5. DAFERMOS, contraction semigroups and trend to equilibrium in continum mechanic. Lecture Notes, $503(1975)$.

6. FRIEDMAN, J. Analyse Math. 11 (1963). 381-413.

7. FRIEDMAN-SHINBROT, Trans. Amer. Math. Soc. 126 (1967), $131-179$.

8. LEVIN, J. Analyse Math. 11 (1963), 381-413.

9. LEVIN, J. Differential Equations 4 (1968) 176-186.

10. MCCAMY-WONG, Stability theorems for some functional differential equations. Trans. A.M.S. 164 $(1972), 1-37$.

11. HALE, Functional Differential Equations. Springer-verlag (1971). 